\title{
INTENSIDAD DE LA MUSICA: EFECTO SOBRE LA FRECUENCIA CARDIACA Y EL ESFUERZO PERCIBIDO DURANTE LA ACTIVIDAD FISICA
}

\author{
Jorge Marín Hernández y Luis Fernando Aragón Vargas \\ Escuela de Educación Física y Deportes \\ Universidad de Costa Rica, San José, Costa Rica \\ E-mail:jgmarín@cariari.ucr.ac.cr
}

\begin{abstract}
Resumen
Marín, J., y Aragón-Vargas, L. F. (2001) Intensidad de la música: efecto sobre la frecuencia cardiaca y el esfuerzo percibido durante la actividad física. Revista de Ciencias del Ejercicio y la Salud, 1(2), 38-42. La música ha sido ampliamente utilizada en la práctica de ejercicios aeróbicos en la industria deportiva. El propósito de este estudio fue comparar el efecto de diferentes intensidades de la música sobre la frecuencia cardiaca (FC) y el esfuerzo percibido (EP) durante ejercicio estandarizado en un cicloergómetro. 13 hombres y 5 mujeres voluntarios, físicamente activos (edad $20.6 \pm 2.2$ años) realizaron una prueba bajo tres condiciones en diferentes días, asignadas en un diseño contrabalanceado de medidas repetidas: no música (NM), música a 70 $d B$, (M70) y música a $85 d B$ (M85). Ellos se ejercitaron por 20 min. con una carga de trabajo constante predeterminada y equivalente al 80\% de su FCMax ( $k p=1.47 \pm 0.4$, $90 \mathrm{rev} / \mathrm{min})$. La música utilizada fue del tipo "Merengue House" en español con un tempo de $131 \pm 4.30 \mathrm{bpm}$, las diferentes intensidades fueron calibradas con un sonómetro. La FC y el EP fueron registrados a los 10 y 20 min. con un monitor de FC Polar y la escala EP-15 de Borg.

\begin{tabular}{ccccc}
\hline Prueba & FC 10 min. & FC 20 min. & EP 10 min. & EP 20 min. \\
\hline NM & $164.78 \pm 8.37$ & $172.11 \pm 8.54$ & $12.06 \pm 1.92$ & $13.78 \pm 2.58$ \\
$M 70$ & $165.11 \pm 11.16$ & $173.00 \pm 12.05$ & $12.00 \pm 2.11$ & $13.56 \pm 2.89$ \\
M85 & $163.00 \pm 7.58$ & $170.17 \pm 7.87$ & $11.50 \pm 1.55$ & $12.94 \pm 2.07$ \\
\hline
\end{tabular}

Tanto para FC como para EP un ANOVA 3 × 2 con medidas repetidas indicó que los promedios a los 20 min. fueron significativamente mayores que a los $10 \mathrm{~min}$. $(p<0.05)$. Las diferencias en FC y EP entre las tres condiciones no fueron significativas ( $p>0.05$ ). Los resultados indican que los rangos de intensidades de música estudiados M70 y M85 no produjeron un efecto sobre el EP y la FC cuando se realizó ejercicio estandarizado en un cicloergómetro comparado con una condición sin música NM. PALABRAS CLAVE: Música, esfuerzo percibido, frecuencia cardiaca, actividad física
\end{abstract}

\section{INTRODUCCION}

Con el surgimiento de los audífonos y la popularidad de los ejercicios aeróbicos en la década de los $80^{\prime}$ s la música se ha convertido en un componente ampliamente utilizado en la industria del acondi-cionamiento físico. Por esto surge la pregunta de cómo este factor puede afectar el rendimiento físico a la hora de practicar un deporte o ejercicio (Smith, 1990). Los efectos psicofísicos de la música se han convertido en un área de gran interés entre los investigadores deportivos de la última década, basando el estudio de la música en el deporte y el ejercicio en tres hipótesis principales: 1) La música desvía la atención lejos de las sensaciones de fatiga. 2) Puede alterar la activación psicomotora y por ello puede ser utilizada como un estimulante $\mathrm{o}$ un tranquilizante previo a la realización de actividad física. 3) Durante la actividad submáxima continua el organismo tiende a responder al elemento rítmico de la música dando como resultado una sincronización entre 
el tempo de la música y la acción del movimiento (Karageorghis \& Terry, 1997)

La música se puede catalogar como un conjunto de factores clasificados como estilo, idioma, ritmo, tempo, melodía, armonía e intensidad (Patton, 1992), que pueden afectar en forma conjunta $\mathrm{o}$ independiente a la hora de interpretar los resultados de las variables a medir. Esto ha provocado resultados contradictorios que imposibilitan tener una conclusión clara de los verdaderos efectos de la música (Karageorghis \& Terry, 1997).

El tratamiento de estos factores como una variable independiente permitiría una mejor compresión de su efecto en variables fisiológicas como la frecuencia cardiaca (FC) y la percepción del esfuerzo realizado a la hora de realizar actividad física. Un factor que puede jugar un rol de mucha importancia es la intensidad o volumen de música utilizado durante una sesión de ejercicio, debido a que:

1) Estudios anteriormente realizados no reportan la intensidad de volumen utilizada, únicamente Copeland y Franks (1991) trabajaron esta como una variable independiente con rangos de 60-70 dB y 75-85 $\mathrm{dB}$ respectivamente, encontrando una disminución significativa en la $\mathrm{FC}$ en unicamente 2 de las 10 mediciones realizadas, cuando los sujetos realizaron ejercicio con una intensidad de 60-70 dB.

2) Como prevención de la salud auditiva de las personas que se ejercitan en gimnasios y centros de salud, la exposición a intensidades superiores a los $90 \mathrm{~dB}$ puede afectar los órganos auditivos (Ganong, 1994), estas lesiones pueden producirse de manera inmediata o suceder al cabo de varios años de continua exposición.

El propósito de este estudio fue determinar el efecto de dos intensidades de la música comparadas con una condición sin música, sobre la frecuencia cardiaca (FC) una variable fisiológica y el esfuerzo percibido (EP) una variable psicofísica durante la realización de actividad física.

\section{METODOLOGIA}

\section{Sujetos}

Participaron en forma voluntaria 18 sujetos (13 hombres y 5 mujeres), estudiantes de los cursos de Actividades Deportivas de la Escuela de Educación Física y Deportes, con edades entre los 18 y 25 años (Tabla 1).

\section{Instrumentos de medición}

- Adaptación del protocolo de Thadem (MacDougall, Wenger \& Green, 1991) para la determinación de la frecuencia cardiaca máxima (FCmax)

- Relojes monitores de frecuencia cardiaca Polar.

- Cicloergómetros Monark E-818

- Tabla de esfuerzo percibido 15 de Borg (Noble \& Robertson, 1996).

- Medidor de sonido (Sonómetro) Radio Shack (cat 33-2050)

- Música: Cinco canciones en idioma español del género "Merengue House" con un tempo de 127 a 135 beats/minuto contenidas en un cassette Maxel fueron tocadas en un Equipo de Sonido Sony FH-150, el cual se encontraba a 2 metros de los sujetos y 1.60 metros del piso.

\section{Procedimientos}

Cada sujeto participó en cuatro sesiones en días diferentes, separados unos de otros por al menos 48 horas. En la primera sesión se determinó la FC Máx, prueba realizada sobre un cicloergómetro, consistiendo de un aumento en la carga de trabajo y la toma de la frecuencia cardiaca respectiva cada 2 minutos hasta alcanzar la fatiga del sujeto o los 14 minutos de duración de la prueba. Las siguientes 3 sesiones consistieron en ejercitarse durante 20 minutos en un cicloergómetro con una carga de trabajo predeterminada $\mathrm{y}$ equivalente al $80 \%$ de su FC $\max$ y 90 revoluciones por minuto (rev/min).

Los sujetos fueron asignados en un orden contrabalanceado de medidas repetidas, a 3 condiciones: A) No Música (NM). B) Música 68-72 dB (M70). C) Música 83-87 dB (M85). 
Los registros de FC y EP se realizaron a la $\operatorname{mitad}(10 \mathrm{~min}) \mathrm{y}$ al final de la prueba $(20 \mathrm{~min})$.

Este estudio se realizó en un laboratorio de ambiente controlado con una temperatura de $23{ }^{\circ} \mathrm{C}$. Durante el estudio a los sujetos no se les informó del objetivo real de medición del efecto de la música, esto se logró indicándoles que el objetivo del estudio era determinar el efecto de diferentes concentraciones de una bebida deportiva, suministrando la misma antes, a los 10 y 20 minutos, una vez finalizada la sesión, durante las 3 pruebas. Se utilizó la misma bebida y la misma concentración en las 3 sesiones y una vez finalizado el estudio se explicó el objetivo real del mismo.

\section{Análisis Estadístico}

Inicialmente se obtuvo la estadística descriptiva, promedios y desviaciones estándar, de las variables edad, talla, peso, Fcmáx y carga de trabajo. Posteriormente se realizó un ANOVA de 2 vías (3 tratamientos, 2 tiempos) con medidas repetidas tanto para FC y EP.

\section{RESULTADOS}

El análisis de los datos por un ANOVA $3 \times 2$ con medidas repetidas para FC indica que los promedios obtenidos en los tres tratamientos NM, M70 y M85 (Fig. 1), no presentan diferencias significativas $F(2,34)$ $=1.70, \mathrm{p}>0.05, \varpi^{2}=0.005$.

Tabla 1.

Características descriptivas del grupo $(\mathrm{n}=18)$. Promedio y Desviación Estándar.

\begin{tabular}{lllllll}
\multicolumn{7}{c}{ Promedio y Desviación Estándar. } \\
& $\begin{array}{l}\text { Edad } \\
\text { Años }\end{array}$ & $\begin{array}{l}\text { Altura } \\
\text { Cm }\end{array}$ & $\begin{array}{l}\text { Peso } \\
\text { Kg }\end{array}$ & $\begin{array}{l}\text { Fcmax } \\
\text { lat/min. }\end{array}$ & $\begin{array}{l}\text { Carga } \\
\text { /Trab kp }\end{array}$ \\
\hline Mujeres & 19.8 & 155.0 & 58.1 & 181.8 & 1.1 & \\
$\mathrm{~N}=5$ & \pm 1.5 & \pm 3.2 & \pm 6.2 & \pm 2.8 & \pm 0.2 & A \\
Hombres & 20.8 & 170.5 & 67.5 & 192.6 & 1.6 & ob \\
$\mathrm{n}=13$ & \pm 2.3 & \pm 6.9 & \pm 9.4 & \pm 8.2 & \pm 0.3 & el \\
& & & & & & \\
Total & 20.6 & 166.2 & 64.9 & 189.6 & 1.47 & $2)$ \\
$\mathrm{n}=18$ & \pm 2.2 & \pm 9.5 & \pm 9.7 & \pm 8.8 & \pm 0.4 & \\
& & & & & & FC
\end{tabular}

Los promedios en la FC obtenidos a los 20 minutos son significativamente más altos
$\mathrm{F}(1,17)=169.25, \quad \mathrm{p}<0.05, \quad \varpi^{2}=0.14$ comparados a los obtenidos a los 10 minutos (Fig. 1).

Figura \# 1.

Promedios de Frecuencia Cardiaca.
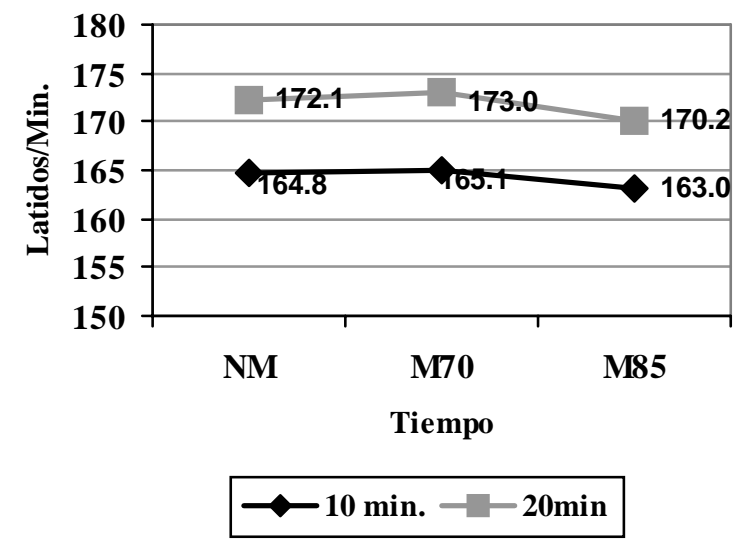

Figura \#2

Promedios de Esfuerzo Percibido

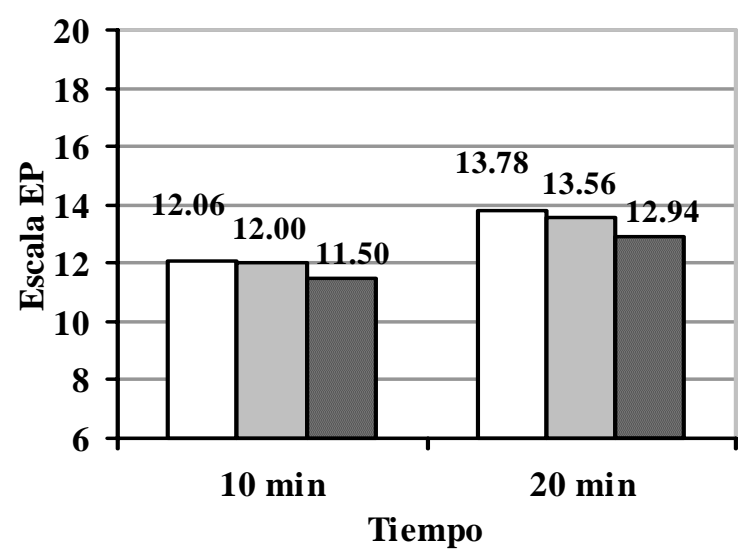

$\square$ Nm $\square$ M70 $\square M 85$

se presenta al comparar los promedios obtenidos en cada uno de los tratamientos para EP a los 20 minutos, los cuales fueron significativamente mayores $\mathrm{F}(1,17)=50.32$, 
$\mathrm{p}<0.05, \omega^{2}=0.11$ con respecto a los obtenidos a los 10 minutos (Fig. 2).

\section{DISCUSION}

Una de las mayores limitaciones en investigaciones previas sobre el efecto de la música en el deporte y el ejercicio ha sido el no especificar las intensidades (volumen) de la música utilizada, complicando la interpretación de los resultados obtenidos debido a que la intensidad o volumen puede influenciar la reactividad y atención hacia la música (Karageorghis \& Terry, 1997).

En la revisión de literatura únicamente los estudios de Copeland y Franks (1991) con tres tratamientos, alta intensidad (75-85 dB), baja intensidad (60-70 dB) y una condición sin música, estudiaron la intensidad de la música como una variable independiente para determinar su efecto sobre la FC y EP.

Al realizar este estudio se esperaba que la música tocada con una mayor intensidad produciría a su vez un efecto distractor mayor en el sujeto, dando como resultado menores registros en la FC a los 10 y 20 minutos en las condiciones con música a M85 como a M70 comparado con la condición NM, manteniendo la misma carga de trabajo, sin embargo los resultados obtenidos no respalda lo anterior, al no presentarse diferencias significativas en los promedios en los tres tratamientos. Estos resultados no son consistentes con los obtenidos por Copeland y Franks (1991) quienes encontraron valores significati-vamente más bajos en la FC cuando los sujetos se ejercitaron con música a baja intensidad (60-70 $\mathrm{dB}$ ) intensidades. Estos resultados no indican un efecto claro de la intensidad de la música, debido a que los promedios estadísticamente más bajos se dieron únicamente en 2 de las 10 mediciones realizadas (Minuto 1 y 6 ).

En el caso de las intensidades altas no se produce una baja en la FC comparado con la condición NM., resultado que es similar al obtenido por Copeland y Franks (1991), esto puede ser explicado por un estudio reciente (Special Report, 1999), que sugiere que la exposición a altas intensidades de música $>90$ $\mathrm{dB}$ produce una situación de estrés, presentándose una elevación de la $\mathrm{FC}$ y la presión sanguínea

A pesar de que no se reporta la intensidad de la música, varios estudios no han encontrado un efecto de la música comparada con una condición control en los registros en la FC. (Patton, 1991), (Lee, Johnson \& Siegel y Schwartz et al, citados en Kargaeorghis \& Terry, 1997).

Con respecto al EP valores más bajos se esperaban a intensidades mas altas de música, previendo un mayor desvío en las sensaciones de fatiga, pero los datos tampoco validan ésta situación, al no encontrarse diferencias significativas en las condiciones con intensidades de música M70 y M85, con respecto a la situación control NM, a los $10 \mathrm{y}$ 20 minutos. Una limitada evidencia al igual que en la FC se encuentra en el estudio de Copeland y Franks (1991) quienes encontraron una diferencia levemente significativa en la condición de música a baja intensidad con respecto a la condición sin música para EP.

En otros estudios donde no se indican las intensidades de la música, no se reporta una baja en la percepción del esfuerzo. En un estudio similar (Abadie et al 1996), no encontró diferencias en el EP cuando los sujetos observaron y escucharon videos comparado con una condición control sin videos, al igual que Patton (1991), al evaluar los efectos de la música familiar y preferida durante la realización de ejercicios aeróbicos.

Al igual que en el caso de la FC, para EP no se produce un efecto positivo en intensidades altas, más bien se sugiere como un factor negativo al considerarse éstas como un factor de estrés, que conlleva a un aumento en los niveles de cortisol, incrementándose la irritabilidad y la fatiga (Special Report, 1999).

Se concluye que las intensidades de música M70 y M85 no producen un efecto sobre la FC y el EP durante la realización de ejercicio estandarizado, comparadas con una condición control sin música NM. Para futuras investigaciones, se debe de evitar la presencia de factores distractores que desvíen la atención hacia la música, en nuestro caso el mantener una determinada cadencia pudo ser ese factor 
distractor, una solución a dicha situación es permitir que los sujetos realicen la prueba con una cadencia voluntaria sincronizados con la música. Basado en lo anterior se podría estudiar el papel de diferentes ritmos, y la interacción con intensidades de música mas altas y su posible efecto sobre el rendimiento, $\mathrm{FC} \mathrm{y} \mathrm{el}$ RPE.

\section{REFERENCIAS}

Abadie, B. Chance,M. O Nan, D \& Lay, C (1996). Viewing music videotapes during exercise and ratings of perceived exertion. Perceptual and motor skills. 83 (1), August: 226,.

Aragón, L.F. \& Fernández, A (1995). Fisiología del Ejercicio. Edit UCR, San José, CR. pp 322.

Azmedra, L. Von Duvellard, S. Le Mura, L \& Aproude, W (1993). Influence of headphone music on cardiovascular hemodinamics, perceived exertion and plasma lactate during treadmill running. Research Quaterly of Exercise and Sport. March: A-36.

Copeland, B \& Franks, B. Effects of types and intensities of background music on treadmill endurance (1991). Journal of Sports Medicine and Physical Fitness. 31 (1), March:100-103.
Editor"S Note. Recommedations for music volume in fitness classes (1997). Idea Today. 15 (6) June: 50.

Ganong, W (1994). Fisiología médica. Edit El Manual Moderno S.A. Mexico: pp 921,

Karageorghis, C y Terry, P.C (1997). The psychophysical effects of music in sport and exercise: a review. Journal of Sport Behavior. 20 (1), March: 54-68.

MacDougall, J. Wenger, H. \& Green, H (Eds) (1991). Physiological testing of the high performance athlete. $2^{\text {nd }}$ Edition. Human Kinetics Publishers. Champaing, IL: pp 143-145.

Noble, B.J. \& Robert, R (1996). Perceived Exertion. Human Kinetics Publishers. Champaing, IL: 320p.

Patton, N (1992). The influence of musical preference on affective state, heart rate and perceived exertion ratings of participants in aerobic dance/ exercise classes. Microform Publications, College of Human Development and Performance, University of Oregon. Thesis.

Smith, K.L (1990). The effects of contemporary rock and roll music on duration, $\mathrm{VO}_{2}$, blood pressure, heart rate and perceived exertion in females aged 18-31 year. Microform Publications, College of Human Development and Performance, University of Oregon. Thesis.

Special Report (1999). Noise. Consumer Reports. September : 19-22, 